\title{
Colgajo miocutáneo de gastrocnemius medial con isla triangular de piel y cierre en $\mathrm{V}-\mathrm{Y}^{*}$
}

\author{
Drs. WILFREDO CALDERÓN O. ${ }^{1}$, VIVIANA SPRÖHNLE F. ${ }^{1}$, ROCÍO LAS HERAS F. ${ }^{2}$, \\ SERGIO LLANOS O. ${ }^{1}$, DANIEL CALDERÓN M. ${ }^{3}$
}

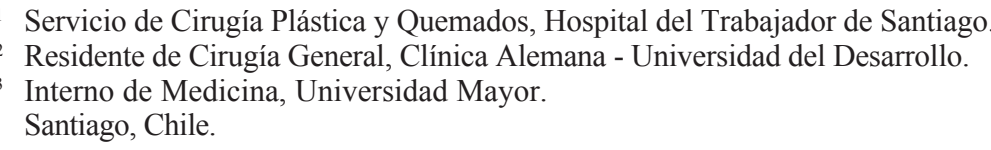

\section{Gastrocnemius muscle myocutaneous flap with a triangular skin isle and V-Y closure. Report of five patients}

A gastrocnemius muscle myocutaneous flap with a triangular skin isle and V-Y closure can be used to correct cutaneous defects with exposure of the proximal part of the tibia. The advantages of this technique are an excellent irrigation and the cutaneous closure using a flap in a zone that is exposed to multiple trauma. The presence of a arteriovenous perforating vessel previously located with color Doppler, ensures the vitality of the flap. We report five males patients aged 20 to 70 years, in whom this technique as used to correct traumatic defects of a mean diameter of $11 \mathrm{~cm}$. All flaps remained viable.

Key words: Gastrocnemius muscle flap, tibia, trauma.

\section{Resumen}

Para corregir defectos cutáneos con exposición de fractura de tibia a nivel del tercio proximal, una buena alternativa es el colgajo miocutáneo de gastrocnemius medial con isla triangular de piel con cierre en V-Y. Las ventajas estriban en la excelente irrigación del colgajo, y en proporcionar un cierre cutáneo con colgajo, en una zona expuesta a múltiples traumatismos. El hecho de tener una perforante arterio venosa previamente ubicada con doppler color, asegura la vitalidad del colgajo. Se presenta la experiencia del Servicio de Cirugía Plástica del Hospital del Trabajador de Santiago consistente en 5 pacientes masculinos en los que se realiza un colgajo de perforante de gastrocnemius medial, con edad entre 20 y 70 años. La etiología del defecto fue traumática. El defecto a corregir fue en promedio de $11 \mathrm{cms}$ de diámetro. Todos los colgajos fueron viables. Se destaca que este es un colgajo inédito, descrito por primera vez por el autor principal en el año 2006.

Palabras clave: Colgajo miocutáneo, gastrocnemius.

\footnotetext{
*Recibido el 13 de Enero de 2009 y aceptado para publicación el 11 de Marzo de 2009.

Correspondencia: Dr. Wilfredo Calderón O.

Ramón Carnicer 201, Santiago, Chile. Fax: 56-2-6853518

E-mail: wcalderon@hts.cl
} 


\section{Introducción}

El colgajo de avance en V-Y fue descrito por primera vez por Esser en $1917^{1}$ y luego se han descrito diversas variaciones ${ }^{2}$. Es un colgajo preferido por numerosos cirujanos, especialmente para el cierre de defectos faciales y de úlceras por presión, ya que es fácil de levantar y la zona dadora tiene baja morbilidad $^{3}$. Además el color y la textura del colgajo son generalmente compatibles con la zona receptora. A pesar de las evidentes ventajas de este colgajo, la movilidad es limitada y el tamaño está predefinido por el tamaño del defecto, como describió Andrades, Calderón, Léniz, Bartel, Danilla y Benítez.

El advenimiento de la microcirugía ha hecho que los colgajos de perforantes cutáneas estén dentro de los predilectos por los cirujanos reconstructivos dadas sus ventajas por sobre los colgajos tradicionales $^{5,6}$. La razón más poderosa para preferir este tipo de colgajo es la mínima morbilidad de la zona dadora, ya que preserva vasos sanguíneos mayores, musculatura e inervación. Es importante destacar también la libertad con la cual se puede diseñar este colgajo, es decir, la capacidad de levantar una paleta cutánea de tamaños variables en cualquier parte del cuerpo que contenga una perforante cutánea apropiada para su irrigación?

Pensando en sumar las ventajas de colgajos de avance en V-Y y de perforantes cutáneas, se han descrito en la literatura colgajos en V-Y basados en perforantes para cubrir variados defectos en extremidades $^{8}$, y en otras localizaciones ${ }^{9}$ con buenos resultados. Se extrapola de la experiencia publicada que el éxito de este colgajo en cualquier parte del cuerpo depende de la elección de una paleta de piel irrigada por una perforante detectable y adecuada.

Considerando la escasez de recursos para cubrir defectos complejos del tercio medio y superior de la pierna, decidimos buscar una perforante cutánea con las características señaladas que nos permitiera avanzar un colgajo miocutáneo de gastrocnemius medial con isla triangular de piel y cierre en V-Y y así proveer una alternativa en el manejo de estos defectos.

\section{Material y Método}

Se realizó un estudio retrospectivo de todos los pacientes en los cuales se hizo un colgajo miocutáneo de gastrocnemius medial con isla triangular de piel con avance en V-Y basado en perforante musculocutánea del gastrocnemio para cubrir defectos del tercio medio y superior de la pierna, en- tre enero de 2005 y septiembre de 2007 en el Servicio de Cirugía Plástica y Quemados del Hospital del Trabajador de Santiago. Todos los pacientes fueron evaluados con ultrasonografía doppler en el estudio preoperatorio, donde fue identificado el vaso perforante (Figura 1) con un flujo adecuado (Figura 2).

Se incluyeron cinco pacientes en el estudio, todos hombres, con un promedio de edad de 48 años (extremos 20 a 70). La etiología fue traumática en todos los casos. El tamaño del defecto fue variable, con un diámetro mayor entre $7 \mathrm{y} 15 \mathrm{~cm}$, promedio de $11 \mathrm{~cm}$. Todas las intervenciones quirúrgicas fueron realizadas por un mismo cirujano. Se evaluó la satisfacción del paciente en la evolución alejada por medio de una encuesta telefónica realizada por un médico del equipo.

Técnica quirúrgica: Frente a una fractura expuesta de tibia en su tercio proximal y medial con una extensión que impide su cierre primario y no permite injerto dermoepidérmico, realizamos un colgajo usando una isla triangular de piel miocutánea.

Al inicio de la operación se talla una isla triangular de piel que queda unida sobre el músculo gastrocnemius medial, cuya base es el borde de la herida (Figura 3).

Posteriormente, se desinserta el gastrocnemius medial del tendón de Aquiles con la isla triangular de piel en su porción superior. A continuación se desplaza, cubriendo completamente la fractura expuesta, suturándolo a los tejidos vecinos. La isla triangular de piel se irriga directamente por perforante muscular (Figura 4). Finalmente, se sutura la piel por planos dejando oclusión cutánea completa (Figuras 5 y 6).

\section{Resultados}

El vaso perforante fue identificado en el intra operatorio con facilidad en todos los casos, encontrándolo en el lugar descrito por la ultrasonografía doppler en el estudio previo. El tiempo operatorio fue en promedio de 1,8 hrs. Todos los colgajos prendieron completamente y evolucionaron de manera habitual. No hubo complicaciones en el postoperatorio inmediato ni alejado. Los pacientes dados de alta fueron evaluados periódicamente por nuestro equipo, encontrándose todos en buenas condiciones, con excelentes resultados funcionales y estéticos (Figuras 3 a 6).

La encuesta telefónica a los pacientes fue positiva, encontrándose todos muy conformes con el resultado de su cirugía. Se tiene seguimiento completo del grupo entre 3 y 34 meses, promedio 13 meses. 


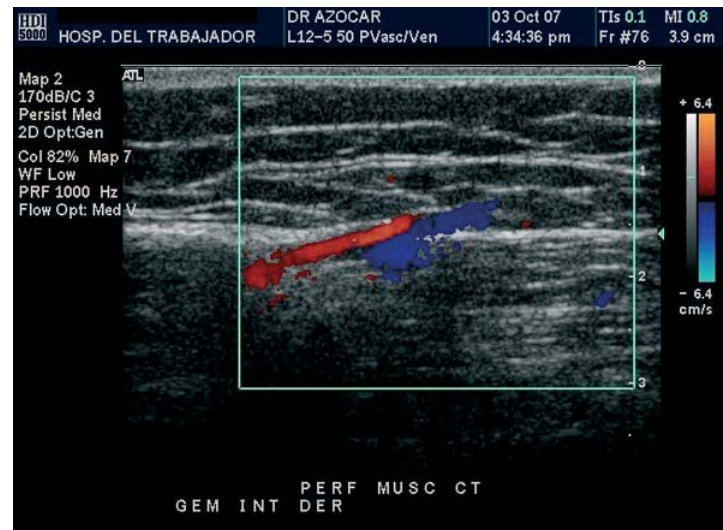

Figura 1. Perforante musculocutánea de gastrocnemio medial.

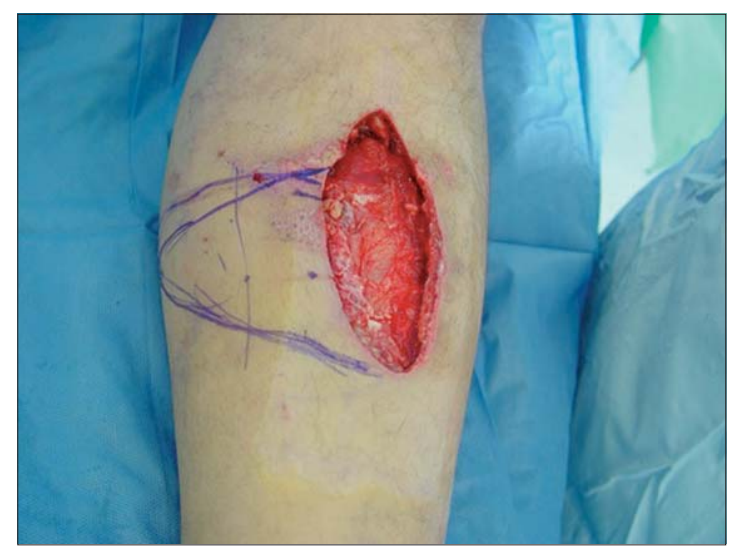

Figura 3. Defecto en tercio medio de la pierna con el colgajo diseñado.

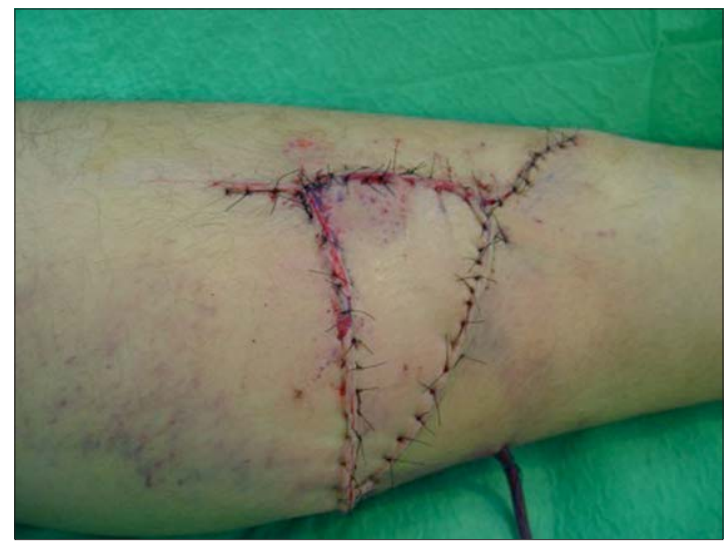

Figura 5. Resultado en el postoperatorio inmediato.

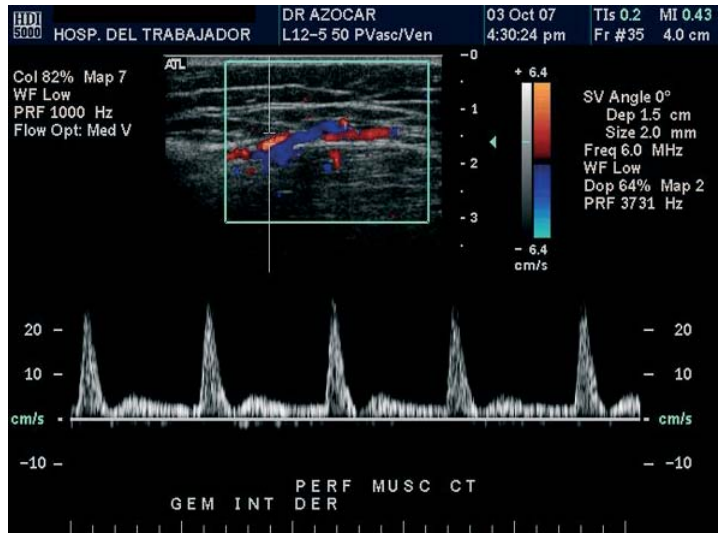

Figura 2. Flujo arterial del vaso perforante musculocutáneo.

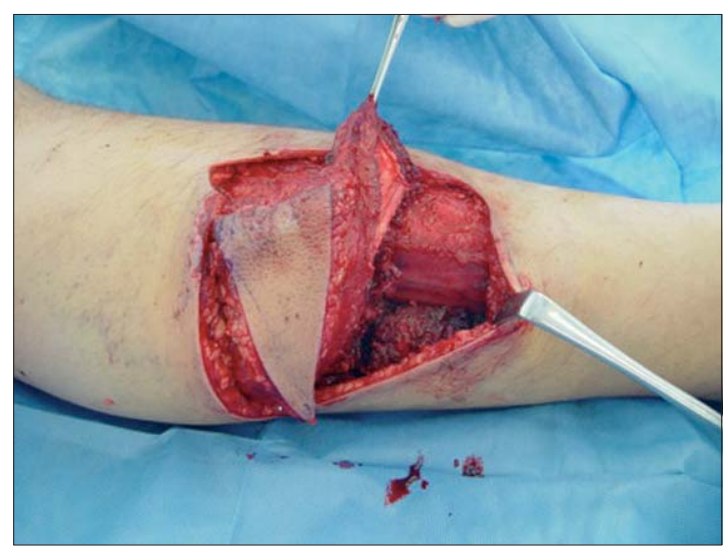

Figura 4. Colgajo de avance en V-Y tallado basado en la perforante de gastrocnemio medial identificada con ultrasonografía.

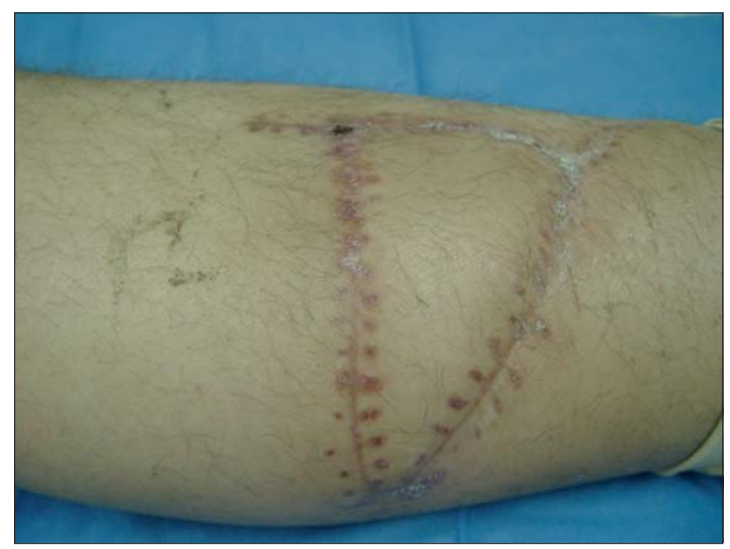

Figura 6. Resultado alejado a 3 meses. 


\section{Discusión}

En 1987 Taylor y Palmer publicaron un estudio anatómico donde describieron más de 300 perforantes cutáneas a lo largo del cuerpo ${ }^{10}$. Al año siguiente, Kroll y Rosenfeld acuñaron el término "colgajos basados en perforantes" en un trabajo donde describieron la utilización de colgajos basados en perforantes cutáneas para cerrar defectos de la línea media posterior ${ }^{11}$. Desde entonces, este tipo de colgajos se ha utilizado ampliamente, sobre todo con el advenimiento de la microcirugía que ha permitido utilizarlo como colgajo libre.

La popularidad de los colgajos de perforantes se debe a la gran versatilidad que ofrecen. Se han convertido en el último tiempo en una excelente alternativa para la cobertura de grandes defectos de las extremidades inferiores dado que ofrecen una vascularización confiable, tienen un amplio rango de movilidad y proporcionan una mayor cantidad de tejido que los colgajos clásicos. Además la morbilidad de la zona dadora es muy baja ya que generalmente se preservan los músculos y los vasos de mayor calibre.

Por otra parte, los colgajos de avance en V-Y han sido una alternativa confiable para la cobertura de defectos pequeños y medianos por décadas. La toma del colgajo es relativamente simple, pero nos hemos visto enfrentados a su movilidad limitada y a su tamaño relativamente pequeño que nos impide utilizarlo para cubrir defectos extensos.

La sumatoria del colgajo en V-Y con el colgajo basado en perforante, ha permitido diseñar un colgajo versátil que ofrece nuevas alternativas para los cirujanos plásticos. Con el colgajo en V-Y basado en perforantes se puede avanzar más distancia y cerrar defectos más grandes que con el de avance por sí sólo y con una morbilidad mínima. Yildirim, Taylan y Aköz incluso han descrito la rotación de este colgajo en $90^{\circ}$ junto con el avance sin ningún compromiso vascular'. Por otra parte, han reportado dificultad inicial en el drenaje venoso del colgajo, especialmente en aquellos de mayor tamaño, o basados en una sola perforante, pero ha sido de resolución espontánea sin llegar ninguno a la necrosis. Otra ventaja muy importante de este tipo de colgajo es que permite cerrar en un mismo tiempo quirúrgico la zona dadora, disminuyendo la morbilidad y mejorando el resultado estético.

En el presente estudio la identificación del vaso perforante se hizo sin dificultad con ultrasonografía doppler en todos los casos. Pudimos observar de manera constante la presencia de una perforante musculocutánea que se origina del músculo gastrocnemio medial y provee irrigación a la piel inmediatamente por sobre ella. Los colgajos de avan- ce en $\mathrm{V}$-Y empleados basándonos en esta perforante proporcionaron una adecuada cobertura para los defectos del tercio medio y superior de la pierna. En el seguimiento alejado mostraron excelentes resultados estéticos y funcionales de la extremidad.

Cabe destacar que este colgajo fue descrito por primera vez por el autor en el año 2006, siendo un colgajo completamente inédito ${ }^{12}$.

En conclusión, nos parece que el colgajo en V-Y basado en perforante de gastrocnemio medial es una muy buena alternativa para el cierre de defectos del tercio superior de la pierna, que permite solucionar un problema complejo en un sólo tiempo quirúrgico y con excelentes resultados a largo plazo.

\section{Referencias}

1. Esser FJS. Island Flaps. NY Med J 1917; 106: 264265.

2. Atasoy E, Ioakimides E, Kasdan ML, Kutz JE, Kleinert HE. Reconstruction of the amputated fingertip with a triangular volar flap. J Bone Joint Surg [Am] 1970; 52: 921-926.

3. Pribaz JJ, Chester CH, Barrall DT. The extended V-Y flap. Plast Reconstr Surg 1992; 90: 275-280.

4. Andrades P, Calderón W, Léniz P, Bartel G, Danilla S, Benítez S. Geometric analysis of the V-Y advancement flap and its clinical applications. Plast Reconstr Surg 2005; 115: 1582-1590.

5. Koshima I, Inagawa K, Yamamoto M, Moriguchi T. New microsurgical breast reconstruction using free paraumbilical perforator adiposal flap. Plast Reconstr Surg 2000; 106: 61-65.

6. Kim DY, Cho SY, Kim KS, Lee SY, Cho BH. Corrections of axillary burns scar contracture with the thoracodorsal perforator based cutaneous island flap. Ann Plast Surg 2000; 44: 181-187.

7. Wei FC, Mardini S. Free style free flaps. Plast Reconstr Surg 2004; 114: 910-916.

8. Venkataramakrishnan V, Mohan D, Villafane O. Perforator based V-Y advancement flaps in the leg. Br J Plast Surg 1998; 51: 431-435.

9. Yildirim S, Taylan G, Aköz T. Freestyle perforatorbased V-Y advancement flap for reconstruction of soft tissue defects at various anatomic regions. Ann Plast Surg 2006; 58: 501-506.

10. Taylor GI, Palmer JH. The vascular territories (angiosomes) of the body. Experimental study and clinical applications. Br Plast Surg 1987; 40: 113-141

11. Kroll SS, Rosenfeld L. Perforator based flaps for low posterior midline defects. Plast Reconstr Surg 1988; 81: 561-566.

12. Calderón W, Llanos S, Rodríguez A, Searle S, Calderón D. Cutaneuos island with V-Y closure over a gastrocnemius muscle flap. Plast Reconstr Surg 2006; 118: $301-302$. 